Artículo

\title{
Áreas verdes urbanas en Córdoba, Veracruz, cantidad, ubicación y acceso: un análisis ortogonal
}

\author{
Gilberto González-Kuk \\ Rafael A. Muñoz-Márquez Trujillo ${ }^{\S}$ \\ J. Cruz García-Albarado \\ Fernando Carlos Gómez-Merino
}

Colegio de Postgraduados-Campus Córdoba-Maestría en Paisaje y Turismo Rural. Carretera Federal Córdoba-Veracruz km 348, Congregación Manuel León, Amatlán de los Reyes, Veracruz, México. CP. 94946. (gilbertogk@outlook.com; jcruz@colpos.mx; fernandg@colpos.mx).

${ }^{\S}$ Autor para correspondencia: arturom@colpos.mx.

\section{Resumen}

Los objetivos de este trabajo fueron registrar las áreas verdes, su localización, superficie y distribución, con respecto de las zonas de alta densidad poblacional, las distancias necesarias para acceder a ellas y la dotación por persona en la ciudad de Córdoba, Veracruz, México. Esto con respecto de los estándares que establece la Organización Mundial de la Salud. Se aplicó un análisis ortogonal, basado en la interpretación de imagen de satélite Google Earth y Sistemas de Información Geográfica. Se hizo además un análisis de distancias a partir de áreas de influencia de cada área verde. Los resultados muestran que las áreas verdes de la ciudad de Córdoba están localizadas sin una aparente relación con las ubicaciones de las zonas de mayor densidad de población, la superficie de áreas verdes es muy heterogénea, la distancia promedio hacia ellas es la recomendada; sin embargo, no hay certeza de que ese destino satisfaga las necesidades de los usuarios, la proporción de área verde por habitante fue de $4.02 \mathrm{~m}^{2}$. Se concluye que la aproximación metodológica utilizada es adecuada para la gestión de área verdeAV a escala urbana. Se concluye también que su superficie y distribución en la ciudad es muy heterogénea; las distancias al área verde más cercana desde cualquier punto, aunque aceptables, no son indicador de tener alcance a un área que satisfaga las necesidades del usuario; la dotación de área verde por habitante en la ciudad está por debajo de la mitad que la OMS recomienda.

Palabras clave: acceso, dotación, espacios abiertos, gestión, SIG.

Recibido: junio de 2019

Aceptado: octubre de 2019 


\section{Introducción}

Las áreas verdes (AV) en las ciudades son importantes de acuerdo con la Organización Mundial de la Salud (OMS) y ésta sugiere una dotación de $9 \mathrm{~m}^{2}$ por habitante (Sorensen et al., 1998; Briceño, et al., 2010). Aunque existen otras recomendaciones (Wang, 2009; Salvador, 2003; Dahl y Molnar, 2003), esta es una referencia importante y ampliamente difundida. La Agenda 2030 para el desarrollo sostenible aprobada en 2015 con la participación de 193 países (entre ellos México), ha planteado en su objetivo 11: 'lograr que las ciudades y los asentamientos humanos sean inclusivos, seguros, resilientes y sostenibles' (ONU, 2015a).

Considerando como un eje de acción la promoción de AV que promuevan una mejor calidad de vida a la ciudadanía y contrarresten los efectos emergentes del cambio climático, entre otros problemas. Por tanto, estas recomendaciones, tienen que ver con diversos aspectos relacionados con el bienestar humano, ya que su presencia en las ciudades, y los servicios ecosistémicos que éstas prestan a las comunidades humanas (y no humanas) (Mendonça y Szlafsztein, 2019), permiten balancear las pérdidas en el bienestar, que la población obtiene como resultado del crecimiento descontrolado y a veces no planeado de la mancha urbana (Muñoz, 2014; ONU, 2015b).

Según la Organización de las Naciones Unidas (ONU), actualmente, 55\% de la población mundial vive en ciudades, y para 2050, aumentará en 13\% (Debnath et al., 2014; ONU, 2018), en este contexto, es necesario pensar en un desarrollo urbano planificado, enmarcado en propuestas sostenibles, lo cual requiere, de una apropiada infraestructura y equipamiento urbano, y entre ellos, una adecuada dotación y distribución de AV (Vélez, 2009). Los espacios públicos, tales como calles, plazas y parques, son necesarios para lograr una sociedad que viva con estándares adecuados para su salud tanto física, social y espiritual (Pérez-Medina y López-Farfán, 2015; Martínez-Soto et al., 2016; OMS, 2017).

De acuerdo con la OMS, existe una clara relación entre las características, ubicaciones y accesos a las AV y la calidad y confort social (OMS, 2017). Esta investigación, analiza la situación que las AV, tienen en la ciudad de Córdoba, Veracruz, México. Trata de entender, cómo éstas se encuentran distribuidas, y el acceso, con respecto de la distancia a los centroides que cada manzana tiene con su área verde más cercana. Este trabajo, se enmarca en una preocupación porque esta ciudad tienda hacia un estado en el cual la población, alcance un estado de bienestar en su desarrollo. Es por ello, que esta investigación se involucra con diversos conceptos que a continuación se describen y conforman el marco teórico en que la misma se desarrolla.

El primer concepto es 'calidad de vida'. La OMS, define a la calidad de vida como 'la percepción del individuo de su situación en la vida, dentro del contexto cultural y de los valores en que vive y en relación con sus objetivos, expectativas, valores e intereses' (Rodríguez, 2012). Se trata de un concepto que involucra las dimensiones físicas, sociales y emocionales (Stokols, 1992). Por otro lado, Ferrer (2008), integra en la calidad de vida, aspectos de satisfacción objetivos y subjetivos, dependientes de cada individuo y sus circunstancias, por lo que todo 'viviente' se realiza dentro de un margen personal y es en dicho margen es donde, de realizarse, encuentra cumplimiento el concepto de calidad de vida que, de inicio es individual. 
En una liga directa entre el espacio urbano y la calidad de vida, Salas-Zapata et al. (2016), puntualizan la importancia del entorno (urbano) ya que se trata de un factor directo entre la calidad de vida de los habitantes de una ciudad y la estrategia de un desarrollo sustentable en ella. Se busca mejorar esos entornos, para convertirlos en condicionantes para disminuir las inequidades sociales. Se puede entonces, comentar que la calidad de vida en las ciudades considera las cualidades del entorno, como un medio que permita a sus habitantes cumplir sus objetivos y expectativas en sus dimensiones físicas, sociales y emocionales.

Con respecto del concepto de 'ciudad sustentable', se debe partir de la definición de desarrollo sustentable, que propone la WCD (1990), como aquel que 'satisface las necesidades del presente sin comprometer las necesidades de las futuras generaciones'. López (2004) indica que el concepto de desarrollo sustentable de inicio no definió explícitamente su posición frente a la urbanización, sin embargo, indica tres elementos importantes para los planificadores: 1) está inscrito en un entorno físico, el del hábitat en todas las escalas; 2) está inscrito en el tiempo, en la historia: tiene que permanecer; y 3) debe inaugurar una nueva era de prosperidad sustentable; es decir, transmisible y patrimonial (Ruano, 2000).

Es hasta la conferencia mundial de hábitat 2 en Estambul y dentro de la Agenda 21, donde se pone de manifiesto el papel de las ciudades y sus gobiernos, en su compromiso al respecto del ambiente global y la consecución de la calidad de vida conveniente, destacando la generación de vivienda adecuada para todos y la consideración del desarrollo sustentable en el diseño y manejo de las ciudades (López, 2004). Flores-Xolocotzi (2017), propone dos dicotomías para entender el desarrollo sostenible en ciudades, siendo la primera el enfoque teórico de racionalidad, y la segunda, el enfoque teórico de lo que se entiende como sustentabilidad (Flores-Xolocotzi, 2017).

En el primer caso, se trata de una orientación hacia un enfoque de racionalidad instrumental y acción racional; es decir, aquella relacionada fuertemente con dominios académicos puramente técnicos, y la acción de estos. En el segundo caso, se trata de tres formas para definir a la sostenibilidad: débil, fuerte y súper fuerte (Maldonado-Villalpando et al., 2018), las cuales tienen un papel preponderante en la construcción de enfoques relacionados con la economía ambiental, la economía ecológica y la economía social y solidaria.

La sostenibilidad súper fuerte considera la valoración múltiple del ambiente y no se constriñe solamente a su valor económico o ecológico, y considera, entre otras, las limitaciones de la ciencia y la tecnología, reconociendo el principio precautorio, y resultando en transformaciones más radicales en contraste con los enfoques de desarrollo convencional (Gudynas, 2011). Con respecto de la sostenibilidad fuerte, ésta considera dimensiones sociales, económicas, políticas y culturales, partiendo del territorio, con el propósito de considerar la complejidad de los socioecosistemas.

Mantiene, asimismo, una relación con la economía ecológica, el enfoque de ecosistemas y el de los sistemas socioecológicos (Maldonado-Villalpando et al., 2018). Por último, la sustentabilidad débil considera los supuestos de la economía ambiental neoclásica con cierto matiz keynesiano (Pierri, 2005). De acuerdo con Flores-Xolocotzi (2017), ésta se representa por la economía ambiental, con un enfoque de racionalidad instrumental, representando una visión de planeación neoclásica. Hace énfasis en los procesos de planeación a corto plazo. Se considera, que la investigación que se presenta en este estudio se enmarca en una sustentabilidad débil, dado que, se trata de un trabajo que considera el valor (no necesariamente de mercado, pero valor), que la sociedad impone hacia las AV y que se muestra en la presencia y distribución actual de las mismas en la traza urbana. 
En cuanto al concepto de 'ciudades inteligentes', Sikora-Fernández (2017) plantea que la ciudad contemporánea no se trata solamente de su estructura física, sino de una gran red de interconexiones que tienen como objetivo la optimización del consumo de los recursos urbanos y procesos de prevención de los efectos negativos como resultado de su funcionamiento, todo ello dentro de la idea de desarrollo sustentable. Este concepto se identifica con el rol de las tecnologías de la información y comunicación (TIC), en el funcionamiento de las ciudades.

Además, Anguluri y Narayanan (2017) indican que es necesario, en un contexto de ciudades sustentables, considerar urbes verdes inteligentes, para poder alcanzar dicho estado. En este marco, el papel de la infraestructura verde (Austin, 2014). En este análisis, la dotación de AV y distancia caminable, son de los conceptos principales de trabajo. La dotación, la definimos como la cantidad de área verde mínima (de acceso público), por habitante $\left(\mathrm{m}^{2}\right.$ hab $\left.{ }^{-1}\right)$, presente en una determinada demarcación urbana. Estas propuestas descansan en los beneficios (servicios ecosistémicos) que estos espacios verdes tienen para la ciudad (Vásquez, 2016; Morales-Cerdas et al., 2018; Yan et al., 2018; Xiao et al., 2018; Vugcic et al., 2019). Flores-Xolocotzi (2017) hace una extensa revisión de estos indicadores. El Cuadro 1 hace un recuento de estos.

\section{Cuadro 1. Dotaciones de área verde (AV) sugeridas por diferentes autores (Flores-Xolocotzi,} 2017).

\begin{tabular}{ccc}
\hline Dotación & Fuente & Observaciones \\
\hline $40 \mathrm{~m}^{2} \mathrm{hab}^{-1}$ & Wang (2009) & No se especifica si son áreas de libre acceso \\
$8{\mathrm{a} 12 \mathrm{~m}^{2} \mathrm{hab}^{-1}}^{2}$ & Salvador (2003) & No se especifica si son áreas de libre acceso \\
$9 \mathrm{~m}^{2} \mathrm{hab}^{-1}$ & ONU (2015b) & \\
$40.5 \mathrm{~m}^{2} \mathrm{hab}^{-1}$ & Dahl y Molnar (2003) & Indican que es un sistema de parques \\
\hline
\end{tabular}

Sin embargo, Garvin (2016) sostiene que las recomendaciones o estándares no generan un sistema adecuado de este tipo de infraestructura. Es la política y liderazgo la que puede generar un sistema más loable de AV en una ciudad, y esto no puede ser sustituido por estándares. Algunas veces estos pueden funcionar en situaciones de baja urbanización y con tierra disponible para ello, pero en áreas donde ya existe desarrollo urbano, esto es más complicado, con lo que coincide la OMS (2017). Respecto a las distancias también existen diversas recomendaciones, como por ejemplo Sorensen et al. (1998), quienes recomiendan una distancia máxima de 15 minutos a pie.

Por otro lado, el ITDP (2017), sugiere una distancia caminable máxima de $500 \mathrm{~m}$ a un parque o zona de juego. Los datos sugeridos son también diferentes, además de que se proponen o en unidades de tiempo, o distancia. La investigación acerca de varios aspectos relacionados con las AV en las ciudades es vasta y se relaciona con diversos temas, que van desde lo ambiental a lo social. A continuación, se enlistan algunos de ellos que se consideran relevantes y relacionados con la investigación que aquí se presenta, enfocados principalmente a México y Latinoamérica.

Ayala-Azcárraga et al. (2019), analizaron a usuarios de nueve parques la Ciudad de México, para determinar los patrones de uso y su efecto en su bienestar. Los autores hallaron una relación muy cercana entre la manera en que los usuarios utilizan esas áreas y los componentes como la distancia, abundancia de arbolado, seguridad, limpieza y calidad del espacio. Morales-Cerdas et al. (2018) desarrollan un estudio que tuvo como objetivo determinar las condiciones ambientales de las AV, utilizando indicadores como herramienta para la gestión urbana en dos ciudades de Costa Rica. 
Como métodos principales se destacan la utilización de imágenes de satélite Rapid Eye, clasificación supervisada y fotointerpretación. Se determinaron 11 indicadores, destacando, el porcentaje de AV públicas y privadas, tipo y tamaño, zonas verdes efectivas per cápita, cercanía de los poblados a ellas y accesibilidad a las mismas. Entre los resultados destacan: $24.6 \mathrm{~m}^{2} \mathrm{hab}^{-1} \mathrm{y}$ $2.7 \mathrm{~m}^{2} \mathrm{hab}^{-1}$ para las dos ciudades respectivamente. Con respecto a la accesibilidad física, determinaron que, en ambos casos, se tuvo una situación media, de acuerdo con el índice desarrollado por los autores para ello. Vargas y Roldán (2018), desarrollan un trabajo en Barranquilla, Colombia, para determinar la relación entre la satisfacción con la vida y la distancia desde el lugar de residencia hasta el parque más cercano.

Los resultados indican que encontraron niveles de satisfacción alto, en los habitantes que se encontraban más cerca de AV, resaltando su importancia dentro de la infraestructura urbana. Rojas et al. (2016), analizan el potencial de la accesibilidad a AV, en las ciudades de Valdivia y Temuco, en Chile. Basaron su trabajo en el análisis de patrones de movilidad diaria y modos de transporte, particularmente caminar, con base en análisis estadístico de la duración de los viajes. Los autores indican que las variaciones en accesibilidad para las dos ciudades se relacionan con edad y género.

Por otro lado, reportan una mejor accesibilidad para Temuco como consecuencia de que los residentes de dicho asentamiento realizan traslados a pie más largos como parte de su forma de movilidad cotidiana. Barros et al. (2015) desarrollan una investigación exploratoria, en cuanto a superficie de las AV del Barrio Potengi, Natal-RN, Brasil, basada en revisiones bibliográficas, consulta en Google Earth y verificaciones en campo. Encuentran que el área verde total es de 2.2 $\mathrm{km}^{2}$, que corresponde a $27.54 \%$ del área del territorio de la región. Además, reportan que la mayor parte de esa superficie se encuentra en plazas y camellones.

Por su parte, Pérez-Medina y López-Farfán (2015) realizan una medición de superficies de arboladas en Mérida, Yucatán, México, y las condiciones que determinan su presencia y distribución, con base en la utilización de imágenes Landsat ETM (30 m de resolución) y recorridos de campo. Este estudio se realizó en cuatro áreas de la ciudad, con tipologías de vivienda específicas en diferentes zonas de esta. A partir, de una clasificación supervisada en una imagen generada de índice de vegetación de diferencia normalizada (NDVI), se extrajo la clase 'arbolado' y se correlacionó con la densidad de vivienda, densidad de población, tipología de vivienda, y etapas de desarrollo, en las zonas de estudio.

Las superficies se obtuvieron; a través, de Google Earth. Los autores encuentran que las AV y la cobertura del estrato arbóreo están vinculadas directamente con las tipologías de vivienda y el mercado inmobiliario, además con los procesos de manejo, gobierno local y participación de la sociedad. Reyes et al. (2010) realizan un análisis de AV en Chile, basándose en métricas de paisaje. Para ello digitalizaron las mismas a partir de fotografía aérea y SIG. Las métricas utilizadas fueron: superficie total, número, índice de fragmento más grande, e índice de cohesión, entre otros.

Para el análisis de accesibilidad, ésta se midió mediante el módulo Network Analyst de ArcGis 9.2, a $300 \mathrm{~m}$ lineales, medidos desde el centroide de cada manzana al centroide del área verde más cercana. Dicha situación se relaciona con el nivel socioeconómico de la población, resultando que los sectores menos pudientes tuvieron menor acceso, que los de mejor situación en ese sentido. 
Con respecto a los trabajos relacionados con la temática de esta investigación en la zona de estudio, éstos son escasos, destacando el trabajo de Olavarrieta (2001), quien analizó la proporción de área verde por habitante en la ciudad de Córdoba, Veracruz, en el año 1998, y determinó una dotación de $1.1 \mathrm{~m}^{2} \mathrm{hab}^{-1}$. Utilizó para ello medios físicos, como análisis de planos de la ciudad y visitas de campo.

De esta revisión, destacan algunos aspectos metodológicos importantes, como la utilización de percepción remota (Google Earth y otras imágenes de satélite y aéreas), la digitalización de los espacios analizados, la relación de datos censales con centroides y distancias entre AV, también a partir de sus centroides, y con las manzanas o zonas habitacionales analizadas. Por otro lado, se reportan diferentes situaciones de dotación, inclusive dispares, teniendo tan poco como $2.2 \mathrm{~m}^{2}$ $\mathrm{hab}^{-1}$, hasta más de 20. El acceso a las AV considera entre otros los usos que una comunidad tiene en sus modos de traslado.

\section{Materiales y métodos}

El estudio se realizó en la ciudad de Córdoba, Veracruz, México, en el verano de 2018. Ésta se localiza entre los paralelos $18^{\circ} 50^{\prime}$ y $19^{\circ} 00^{\prime}$ de latitud norte; los meridianos $96^{\circ} 52^{\prime}$ y $97^{\circ} 01^{\prime}$ de longitud oeste y con una altitud promedio de $900 \mathrm{~m}$. Tiene un clima cálido húmedo con abundantes lluvias en verano (gobierno del estado de Veracruz, 2018). Este análisis consideró solo la cabecera municipal.

Los objetivos de este estudio fueron: a) identificar y cuantificar la ubicación de las AV públicas en la ciudad; b) determinar su distribución; c) verificar su coincidencia o no, con la localización de las concentraciones de población (áreas de mayor o menor densidad poblacional); d) determinar las distancias que existen desde cada punto de la cabecera municipal a su AV más cercana; e) obtener el dato de la dotación de AV públicas para la población de esa urbe; y d) analizar los resultados obtenidos con respecto de la recomendación de la OMS.

El análisis ortogonal para analizar el tamaño y la localización de las áreas estudiadas consistió en determinar en una superficie horizontal imaginaria, las proyecciones de los contornos o linderos que delimitan a esas áreas. Estos se digitalizaron en un SIG. Se utilizó Google Earth, como base para esa tarea. A partir de ello fue posible determinar la ubicación georreferenciada de esos espacios y sus tamaños. Se seleccionaron AV de acceso libre, excluyendo áreas privadas. Sólo se incluyeron parques públicos, plazas y zonas deportivas. La elección de estas se basó en los criterios mostrados en el Cuadro 2.

Cuadro 2. Áreas verdes (AV) de la ciudad de Córdoba, Veracruz, México en este estudio.

Tipología y aspectos considerados en la elección de AV en el estudio

a) AV (parques) con más del 50\% de cobertura vegetal, sobre áreas pavimentadas

b) AV (parques con pavimento) con menos del 50\% de cobertura vegetal, sobre áreas pavimentadas

c) AV deportivas, para la práctica de deportes diferentes al futbol soccer (baseball, básquetbol, entre otras)

d) canchas para práctica de futbol soccer 
Los criterios del Cuadro 2 arrojaron 34 espacios (Figura 1), a través de la interpretación de imagen de satélite Google Earth $^{\odot}$ (fecha 5/7/2016), con base en aspectos de textura y tono. Para el análisis ortogonal, se construyó un SIG, con QGIS ${ }^{\circledR}$ ver. 2.16.0. Se consideró la cartografía geoestadística urbana, desarrollada por el INEGI (INEGI, 2017a). Dicha cartografía está disponible en formato vectorial. La proyección de esa información se basa en los parámetros ITRF_1992_Datum: D_ITRF_ 1992. Se trabajó con información de áreas geoestadísticas básicas (AGEB) (INEGI, 2017b).
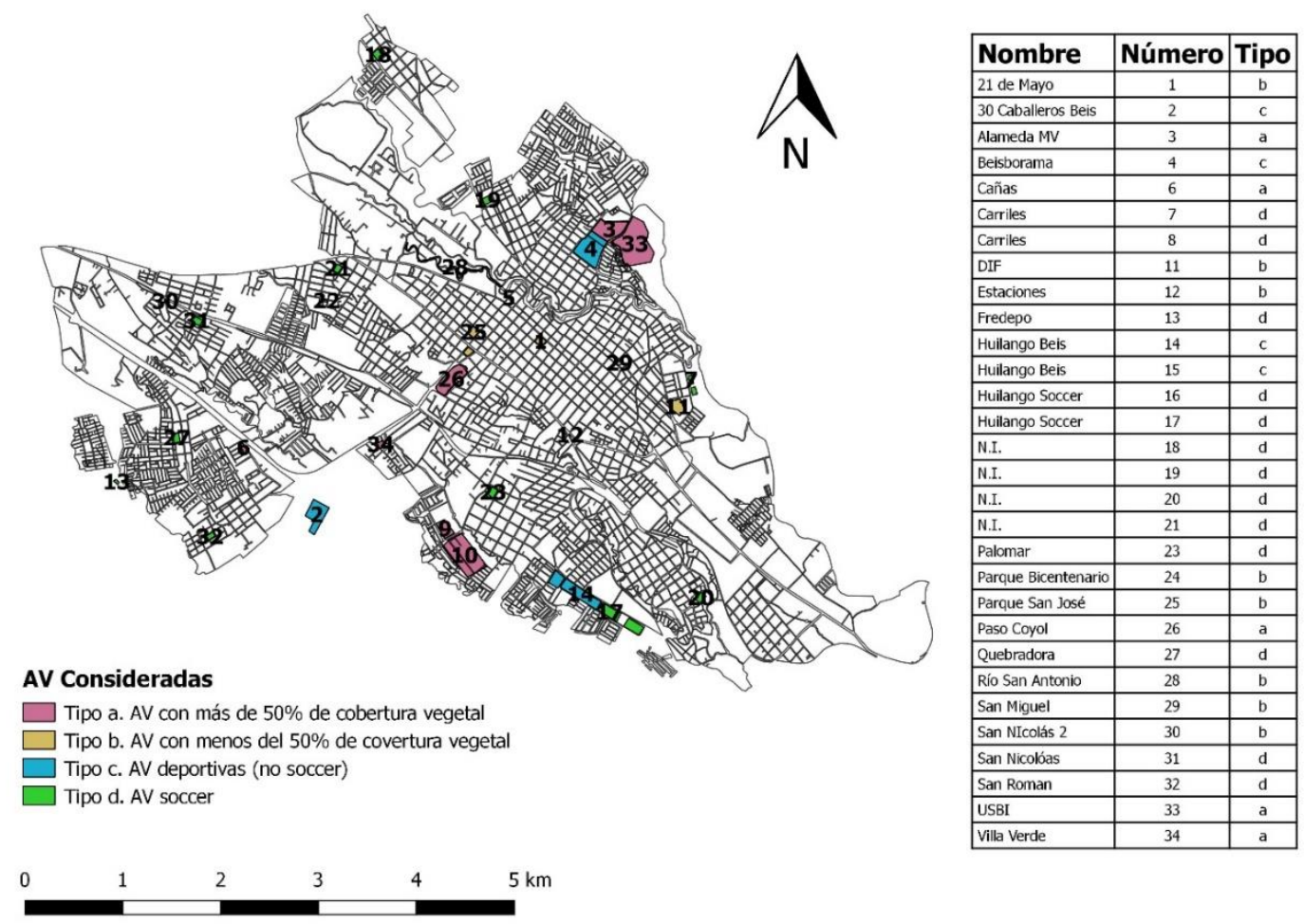

Figura 1. Áreas verdes (AV) identificadas en la ciudad de Córdoba, Veracruz, México mediante imágenes de satélite Google $\operatorname{Earth}^{\odot}$.

Por medio de procesos de unión entre tablas de atributos y archivos vectoriales, se enlazó la información de AGEB, con cada manzana de la ciudad, de manera que se calculó la población presente en cada una de ellas, esto se realizó con base en la determinación de los centroides de cada manzana. A partir de ello, y por procesos geoestadísticos se determinaron las densidades de la población, generando mapas formato ráster en donde se aprecian esas concentraciones. Asimismo, se cuantificaron las superficies de cada AV, y la población que sirve cada una, de acuerdo con la determinación su área de influencia, por medio de un análisis de polígonos de Thiessen (o diagramas de Voronoi) (Tabios y Salas, 1985).

Finalmente se determinó un ráster de distancias de todos los puntos dentro de cada área de influencia a su AV más cercana. A partir de ello, se generó información de disponibilidad, ubicación, distribución en la ciudad, y distancia a las mismas. Con los datos colectados, se realizó un análisis estadístico exploratorio. Los resultados arrojaron diversos mapas y datos relacionados con los objetivos planteados. Se realizaron también recorridos de campo para verificar la totalidad de las AV analizadas, dado que el tamaño relativamente pequeño de la ciudad lo permitió. 


\section{Resultados y discusión}

\section{Ubicación de AV con respecto de la densidad poblacional}

El análisis muestra que la disposición de los 34 espacios no coincide con las ubicaciones de las áreas de mayor densidad poblacional. Existe alguna coincidencia al norte de la zona urbana (Figura 2). Esto sugiere una inadecuada planificación en la ubicación de ellas como equipamiento urbano para la ciudad. La superficie total encontrada de AV $\left(667044.72 \mathrm{~m}^{2}\right)$ no está dispuesta de manera homogénea, como lo sugiere el valor de la varianza del Cuadro 3 existen áreas tan pequeñas como $88.71 \mathrm{~m}^{2}$, así como otras de $125496.27 \mathrm{~m}^{2}$, con alta variabilidad en superficie.

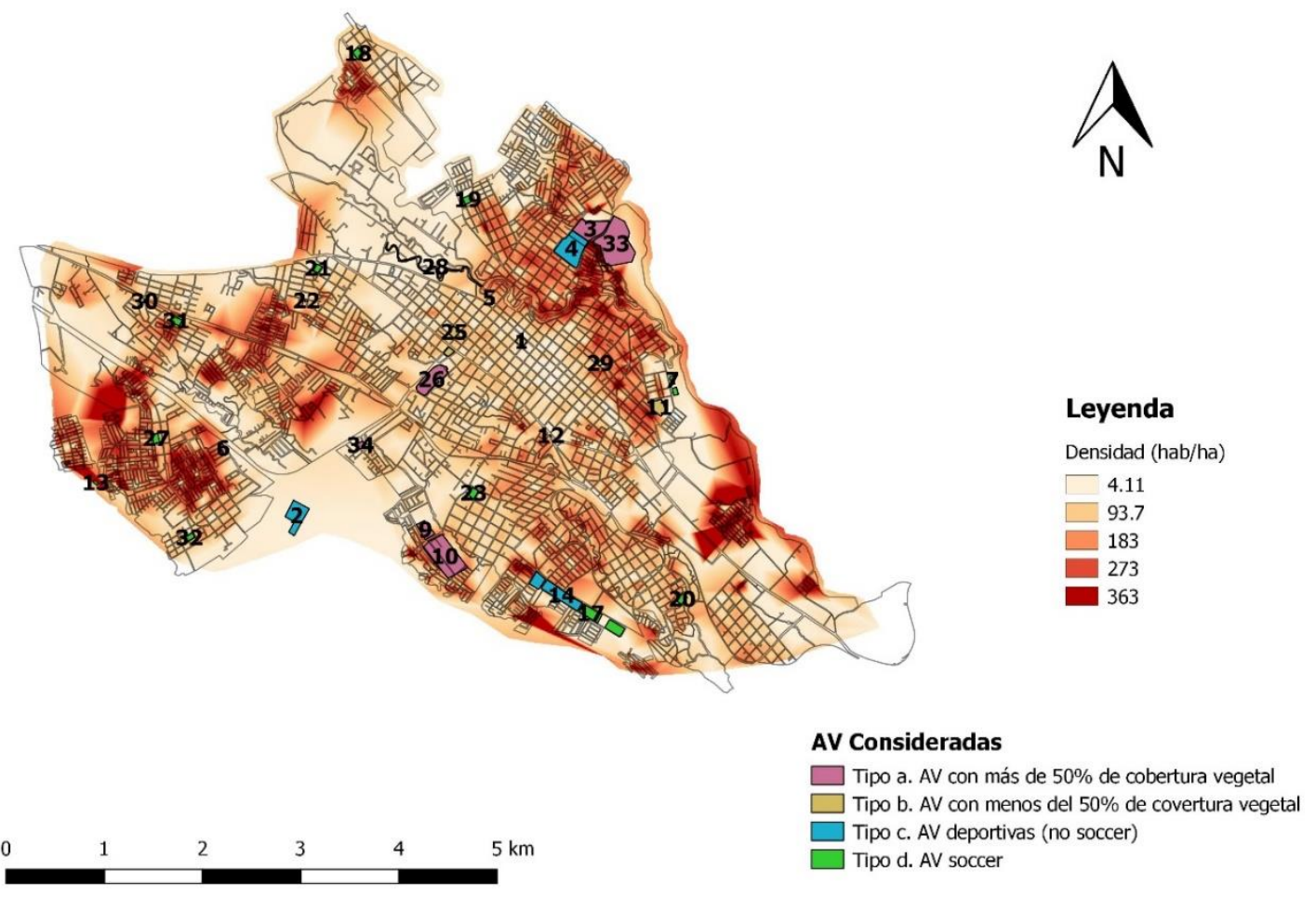

Figura 2. Mapa que representa las áreas verdes (AV) y las densidades poblacionales en la ciudad de Córdoba, Veracruz, México.

Cuadro 3. Estadísticos generales obtenidos por el proceso ortogonal de análisis de áreas verdes (AV) en la ciudad de Córdoba, Veracruz, México.

\begin{tabular}{llcccc}
\hline $\begin{array}{c}\text { Nombre del parque y } \\
\text { área vede }\end{array}$ & $\begin{array}{c}\text { Superficie de } \\
\mathrm{AV}\left(\mathrm{m}^{2}\right)\end{array}$ & $\begin{array}{c}\text { Población total } \\
\text { que sirve cada } \\
\mathrm{AV}\end{array}$ & $\begin{array}{c}\text { Área de influencia } \\
\text { de cada polígono } \\
\text { Voronoi }\left(\mathrm{m}^{2}\right)\end{array}$ & $\begin{array}{c}\text { Distancia media al } \\
\text { parque más cercano } \\
\text { (m lineales) }\end{array}$ \\
\hline 1 & 21 de mayo & 7186.05 & 4651 & 638801.08 & 322.51 \\
2 & 30 caballeros Beis & 41521.85 & 71 & 835456.60 & 369.63 \\
3 & Alameda MV & 48220.28 & 8695 & 844838.37 & 577.34 \\
4 & Beisborama & 69060.93 & 8592 & 601419.48 & 353.42 \\
5 & Bolsillo & 88.71 & 4754 & 470471.22 & 289.35 \\
\hline
\end{tabular}




\begin{tabular}{|c|c|c|c|c|c|}
\hline & $\begin{array}{c}\text { Nombre del parque y } \\
\text { área vede }\end{array}$ & $\begin{array}{l}\text { Superficie de } \\
\qquad \mathrm{AV}\left(\mathrm{m}^{2}\right)\end{array}$ & $\begin{array}{c}\text { Población total } \\
\text { que sirve cada } \\
\text { AV }\end{array}$ & $\begin{array}{c}\text { Área de influencia } \\
\text { de cada polígono } \\
\text { Voronoi }\left(\mathrm{m}^{2}\right)\end{array}$ & $\begin{array}{c}\text { Distancia media al } \\
\text { parque más cercano } \\
\text { (m lineales) }\end{array}$ \\
\hline 6 & Cañas & 4642.6 & 6924 & 1126987.83 & 289.15 \\
\hline 7 & Carriles & 3260.3 & 2135 & 327348.02 & 444.79 \\
\hline 8 & Carriles2 & 3486.4 & 42 & 388994.48 & 345.15 \\
\hline 9 & Cementerio J. de Paz & 17734.9 & 765 & 574574.96 & 605.49 \\
\hline 10 & Cementerio municipal & 97883.99 & 2643 & 514997.83 & 344.09 \\
\hline 11 & DIF & 15184.07 & 5024 & 1112270.98 & 314.94 \\
\hline 12 & Estaciones & 100.97 & 9845 & 1242835.32 & 595.3 \\
\hline 13 & Fredepo & 1372.3 & 5982 & 759908.55 & 458.96 \\
\hline 14 & Huilango Beis & 41899.14 & 6605 & 801607.15 & 485.4 \\
\hline 15 & Huilango Beis2 & 15345.55 & 3799 & 487949.97 & 432.93 \\
\hline 16 & Huilango Soccer & 17892.52 & 1542 & 367019.06 & 414.2 \\
\hline 17 & Huilango Soccer2 & 20204.74 & 1229 & 540334.40 & 383.4 \\
\hline 18 & N.I.1 & 6957.01 & 3514 & 1468680.12 & 339.8 \\
\hline 19 & N.I.2 & 6263.37 & 3863 & 1420029.24 & 638.95 \\
\hline 20 & N.I.3 & 5769.20 & 14981 & 3797998.34 & 574.9 \\
\hline 21 & N.I.4 & 4392.32 & 4058 & 1206046.60 & 1003.92 \\
\hline 22 & Nuevo Córdoba & 463.69 & 8253 & 1218086.59 & 595.48 \\
\hline 23 & Palomar & 6270.91 & 6725 & 868905.46 & 496.44 \\
\hline 24 & Parque bicentenario & 5582.72 & 1706 & 277071.03 & 375.53 \\
\hline 25 & Parque San José & 7457.88 & 1759 & 307595.60 & 413.67 \\
\hline 26 & Paso Coyol & 46160.03 & 3849 & 844005.99 & 432 \\
\hline 27 & Quebradora & 5669.11 & 9003 & 987800.63 & 391.92 \\
\hline 28 & Río San Antonio & 23330.27 & 1339 & 829338.78 & 283.22 \\
\hline 29 & San Miguel & 1592.95 & 10632 & 782171.33 & 351.92 \\
\hline 30 & San Nicolás & 6689.35 & 7256 & 1007503.27 & 686.78 \\
\hline 31 & San Nicolás 2 & 253.48 & 4372 & 1556109.30 & 443.67 \\
\hline 32 & San Román & 5377.38 & 4003 & 806365.27 & 347.47 \\
\hline 33 & USBI & 125496.27 & 2195 & 516822.25 & 343.02 \\
\hline 34 & Villa Verde & 4233.48 & 5018 & 1247095.37 & 441.41 \\
\hline & Totales & 667044.72 & 165824 & 30777440.47 & 15186.15 \\
\hline & Media & 19618.96 & 4877.18 & 905218.84 & 446.65 \\
\hline & Varianza & $833,115,243.9$ & $11,541,703.18$ & $384,630,826,936.14$ & 21587.72 \\
\hline & Mínimo & 88.71 & 42 & 277071.03 & 283.22 \\
\hline & Máximo & 125496.27 & 14981 & 3797998.34 & 1003.92 \\
\hline
\end{tabular}


Si como ya se ha indicado antes, la OMS recomienda que la distancia máxima caminable a un espacio como los analizados no debe de ser de más de 15 minutos caminando (Tabios y Salas, 1985; Sorensen et al., 1998) y si se considera una velocidad promedio de $5 \mathrm{~km} \mathrm{~h}^{-1}$, la distancia que se puede recorrer en ese tiempo resultaría en $1250 \mathrm{~m}$.

Para recorrer $446.65 \mathrm{~m}$, a una velocidad de $5 \mathrm{~km} \mathrm{~h}^{-1}$, el tiempo requerido de caminata sería de alrededor de $5.5 \mathrm{~min}$, lo cual indicaría que, en promedio, las distancias encontradas, serían aceptables. Sucede lo mismo, con la recomendación de ITDP (2017). Sin embargo, como ya se ha mencionado, no se involucran las características físicas o estructurales de las AV destino, por lo que esa distancia no necesariamente garantiza el acceso a una que satisfaga las necesidades de un usuario en términos del uso de ese espacio. Esta información señala una debilidad en la oferta de acceso de manera equitativa a esas áreas. Asimismo, considerando el papel de estos espacios en el bienestar la calidad de vida de los habitantes, la situación analizada no resulta positiva en un marco de sustentabilidad urbana (Flores-Xolocotzi, 2017).

\section{Dotación de AV por habitante}

Los resultados del análisis ortogonal, se muestran en el Cuadro 44. Se puede apreciar que los datos indican una dotación de $4.02 \mathrm{~m}^{2} \mathrm{hab}^{-1}$ de AV para la ciudad. Esto significa un área mayor a la encontrada en 1998 por Olavarrieta (2001). Sin embargo, ésta aún se encuetra por debajo de la mitad del área recomendada por la OMS.

Cuadro 4. Números resultantes del análisis ortogonal de áreas verdes (AV) en la ciudad de Córdoba, Veracruz, México.

\begin{tabular}{cc}
\hline \multicolumn{2}{c}{ Resultados generales } \\
\hline Población total (habitantes) & 165824 \\
Área verde $\left(\mathrm{m}^{2}\right)$ & 667044.72 \\
Área verde/habitante $\left(\mathrm{m}^{2}\right)$ & 4.02 \\
\hline
\end{tabular}

El análisis presentado es ortogonal; es decir, se trabajó sobre la proyección de los límites de los espacios analizados en un medio horizontal; por lo tanto, los datos encontrados corresponden a esa proyección y no a la realidad tridimensional de las AV. Sin embargo, este tipo de análisis es útil como herramienta exploratoria, para la planificación y el manejo de ellas. El levantamiento físico de cada área, que incluya, entre otros aspectos, su planimetría y altimetría, diseño, composición florística, orientación preferencia y uso de esos espacios, es un trabajo pendiente.

En 25 de enero de 2017, se publicó en la Gaceta del Estado de Veracruz, el Plan Municipal de Desarrollo Urbano de la Ciudad de Córdoba, Veracruz (Gobierno del Estado de Veracruz, 2017). Relativo a la dotación y acceso a AV de la ciudad, indica en su diagnóstico, un estado deficitario. Aunque no se indica la metodología empleada, manifiesta, entre otros aspectos, una muy baja dotación de espacios abiertos, y problemas de acceso igualitario. Establece como estrategias, una serie de acciones que las autoridades deben realizar para solventar la situación OMS, e ITDP (2017). 
Debe cumplir con 1) ser abiertos a cualquier usuario; 2) ofrecer algo para cada quién; 3) atraer y retener las demandas del mercado; 4) proporcional un marco para la urbanización exitosa; 5) mantener un ambiente habitable; y 6) nutre y apoya a la sociedad civil. Cabe preguntarse si con la situación encontrada en esta investigación, las AV cumplen en algún grado con esas 6 propuestas, para comprender si se ofrecen alternativas para una mejora en el nivel de vida de las personas. La respuesta parece ser que no, para la colectividad.

Esto mueve a considerar la acción para intentar solventar la situación para comenzar a actuar hacia una ciudad que tenga un sistema de AV menos fragmentado. Ello permitirá avanzar en la consecución de elementos hacia una sustentabilidad. Será necesario tender hacia una sustentabilidad súper fuerte, a fin de valorar la situación de una manera comprensiva y propiciar cambios radicales.

\section{Conclusiones}

La ubicación territorial de las AV en Córdoba, Veracruz, no presenta una distribución que coincida con las concentraciones de la población en la ciudad, lo cual puede generar ineficiencias el en uso y acceso a las mismas, además de inequidad social. La dotación de AV por habitante encontrada es inferior al recomendado por organismos internacionales como la OMS. Aunque la misma organización sugiere que en realidad no hay bases para considerar el indicador de $9 \mathrm{~m}^{2}$ como válido, se puede comentar que la presencia de este tipo de áreas en la ciudad es adecuada en tiempos de cambio global, como elemento de adaptación al mismo, en un contexto de desarrollo urbano sustentable.

La distancia encontrada para acceder a una AV más cercana, desde cualquier punto de su zona de influencia, es adecuada de acuerdo con lo recomendado por la OMS y la ITDP (2017). Sin embargo, esto no indica que dicha AV necesariamente satisfaga las necesidades que determinados usuarios requieran de ella. Este análisis establece bases, para entender la forma en la cual las autoridades y la sociedad valoran y gestionan sus AV. Claro queda que la situación actual en Córdoba no apunta hacia una sustentabilidad urbana.

\section{Literatura citada}

Anguluri, R. and Narayanan, P. 2017. Role of green space in urban planning: Outlook towards smart cities. Urban Forestry \& Urban Greening. 25:58-65.

Austin, G. 2014. Green Infrastructure for landscape planning. Integrating Human and Natural Systems. Routledge. New York. United States of America. 4-5 pp.

Ayala-Azcárraga, C.; Diaz, D. and Zambrano L. 2019. Characteristics of urban parks and their relation to user well-being. Landscape and Urban Planning. 189(9):27-35.

Barros, L. H. V.; Silva, V. P.; Alves, G. A.; Pinheiro, L. G.; Nascimento, I.; Dias, P. K. L.; Oliveira, R. F. A.; Santana, V. A.; Oliveira, W. A.; Medeiros, C. S. C. e Gomes, B. S. F. F. 2015. Diagnóstico das AV públicas do Bairro Potengi, Natal-RN. Holos. 5(5):130-141.

Briceño, M.; Gil, S. B.; Gómez, R. L.; Contreras, M. W. y Owen, C. M. 2010. La ecología urbana y el conocimiento científico. Ecodiseño y Sostenibilidad. 2(1):373-395.

Dahl, B. and Donald, M. 2003. Anatomy of a park. Waveland Press Inc. Illinois. United States of America. 1-13 pp. 
Debnath, A. K.; Chin, H. C.; Haque, M. M. and Yuen, B. 2014. A methodological framework for benchmarking smart transport cities. Cities. 37(2):47-56.

Ferrer-Santos, U. 2008. El viviente, la vida y la calidad de vida. Cuadernos de Bioética. 23(2):213-221.

Flores-Xolocotzi, R. 2017. Una reflexión teórica sobre los estándares de AV empleados en la planeación urbana. Sociedad, Economía y Territorio. 17(54):491-522.

Garvin, A. 2016. What makes a great city. Island Press. Washington. United States of America. $11-18 \mathrm{pp}$.

Gobierno del estado de Veracruz. 2017. Programa Municipal de Desarrollo Urbano de Córdoba, Ver. Gaceta Oficial. 25 de enero de 2017. Tomo CXVC. 36(25):1-244.

Gobierno del Estado de Veracruz. 2018. Cuadernillos municipales 2018. http://www.veracruz.gob.mx/wp-content/uploads/sites/2/2015/05/C\%C3\%B3rdoba.pdf.

Gudynas, E. 2011. Desarrollo y sustentabilidad ambiental: diversidad de posturas, tensiones persistentes. In: Matarán, R. A. y López, C. F. (Eds). La Tierra no es muda: diálogos entre el desarrollo sostenible y el postdesarrollo. Universidad de Granada. España. 80 p.

INEGI. 2017a. Productos. Cartografía geoestadística urbana. Cierre de los Censos Económicos 2014, DENUE 01/2015, Veracruz de Ignacio de la Llave. http://www3.inegi.org.mx/sistemas/biblioteca/ficha.aspx?upc=702825004441.

INEGI. 2017b. Censos y Conteos de Población y Vivienda. Censo de Población y Vivienda 2010, Principales resultados por AGEB y manzana urbana. http://www.inegi.org.mx/sistemas/consulta_resultados/ageb_urb2010.aspx?c=28111.

IDT. 2017. Institute of Transportation and Development Policy. DOT Estándar. ITDP. $3^{\text {a }}$ Edition. New York. United States or America.

Maldonado-Villalpando, E.; Herrera, T. H. A. y Guerrero G. R. H. R. 2018. Diseño de política ambiental e innovación social. Aportaciones teórico-metodológicas para la gestión sustentable de áreas naturales protegidas. Econ. Soc. 22(38):111-128.

Martínez-Soto, J.; Montero, L. M. y De la Roca Chiapas, J. M. 2016. Efectos psicoambientales de las áreas verdes en la salud mental. Rev. Interam. Psicol. 50(2):204-214.

Mendonça, D. C. R. and Szlafsztein, C. F. 2019. Urban vegetation loss and ecosystem services: the influence on climate regulation and noise and air pollution. Environmental Pollution. 245(2):844-852.

Morales-Cerdas, V.; Piedra, C. L.; Romero, V. M. y Bermúdez R. T. 2018. Indicadores ambientales de áreas verdes urbanas para la gestión en dos ciudades de Costa Rica. Rev. Biol. Trop. 66(4):1421-1435.

Muñoz, R. M. 2014. Accesibilidad a las áreas verdes urbanas como espacios públicos. El caso de Ciudad Juárez, Chihuahua. Tesis de Maestría en Acción Pública y Desarrollo Social. Ciudad Juárez, Chihuahua: El Colegio de la Frontera Norte. Ciudad Juárez, Chihuahua, México. 37 p.

Olavarrieta, M. R. 2001. La importancia de las áreas verdes urbanas (AVU) en el desarrollo urbano sostenible de la Ciudad de Córdoba, Veracruz. Tesis de Maestría en Gestión y Promoción Urbana para el Desarrollo Sostenible. Córdoba Veracruz. Facultad de Arquitectura, Universidad Veracruzana. Córdoba, Veracruz, México. 134 p.

OMS. 2017. Organización Mundial de la Salud. Urban green spaces: a brief for action. http://www.euro.who.int/_data/assets/pdf_file/0010/342289/Urban-green-spaces_en_who_ web3.pdf. 
ONU. 2018. Organización de las Naciones Unidas. Las ciudades seguirán creciendo, sobre todo en los países en desarrollo. https://www.un.org/development/desa/es/news/population/2018world-urbanization-prospects.html.

ONU. 2015a. Organización de las Naciones Unidas. Objetivos de Desarrollo Sostenible. Objetivo 11. Lograr que las ciudades y los asentamientos humanos sean inclusivos, seguros, resilientes y sostenibles. https://www.un.org/sustainabledevelopment/es/cities/.

ONU. 2015b. Organización de las Naciones Unidas. Hábital III. Nueva York: Organización de las Naciones Unidas. http://habitat3.org/wp-content/uploads/issue-paper-11_public_spacesp.pdf.

Pérez-Medina, S. y López-Farfán, I. 2015. Áreas verdes y arbolado en Mérida, Yucatán. Hacia una sostenibilidad urbana. Econ. Soc. Territ. 15(47):1-33.

Pierri, N. 2005. Historia del concepto de desarrollo sustentable. In: Foladori, G. y Pierri, N. (Coords.). ¿Sustentabilidad? Desacuerdos sobre el desarrollo sustentable. Miguel Ángel Porrúa, Ciudad de México, México. 27-81 pp.

Reyes, P. S. y Figueroa A. I. 2010. Distribución, superficie y accesibilidad de las áreas verdes en Santiago de Chile. EURE. 36(109):89-110.

Rodríguez, A. E. 2012. Calidad de vida y percepción de salud en mujeres de mediana edad. Invest. Medicoquir. 4(1):245-259.

Rojas, C.; Páez, A.; Barbosa, O. and Carrasco, J. 2016. Accessibility to urban green spaces in Chilean cities using adaptive thresholds. J. Transport Geog. 57(8):227-240.

Ruano, M. 2000. Eco Urbanismo. Entornos urbanos sostenibles: 60 Proyectos. Gustavo Gili. Barcelona, España. 192 p.

Salas-Zapata, L.; López-Ríos J. M.; Gómez-Molina, S.; Franco-Moreno, D. y Martínez-Herrera, E. 2016. Ciudades sostenibles y saludables: estrategias en busca de la calidad de vida. Rev. Fac. Nac. Salud Pública. 34(1):105-110.

Salvador, O. P. J. 2003. La Planificación Verde en las Ciudades. Gustavo Gili. España. 326 p.

Sikora-Fernández, D. 2017. Factores de desarrollo de las ciudades inteligentes. Rev. Univ. Geog. 26(1):135-152.

Sorensen, M.; Barzetti, V.; Keipi, K. y Williams, J. 1998. Manejo de las áreas verdes urbanas. Banco Interamericano de Desarrollo. Washington, DC, Estados Unidos de América. 4 p.

Stokols, D. 1992. Environmental quality, human development, and health: an ecological view. J. Appl. Develop.1 Psychol. 13(2):121-124.

Tabios, G. Q., y Salas, J. 1985. A comparative analysis of techniques for spatial interpolation of precipitaton. J. Am. Water Res. Assoc. 21(3):365-380.

Vargas, A. y Roldán, P. 2018. Ni muy cerca ni muy lejos: parques urbanos y bienestar subjetivo en la ciudad de Barranquilla, Colombia. Lecturas de Economía. 88(2):183-205.

Vásquez, A. E. 2016. Infraestructura verde, servicios ecosistémicos y sus aportes para enfrentar el cambio climático en ciudades: el caso del corredor ribereño del río Mapocho en Santiago de Chile. Rev. Geog. Norte Grande. 63(1):63-86.

Vélez, R. L. 2009. Del parque urbano al parque sostenible. Bases conceptuales y analíticas para la evaluación de la sustentabilidad de parques urbanos. Revista de Geografía Norte Grande. 43(1):31-49.

Vugcic, M.; Tomicevic-Dubljevic, J.; Zivojinovic, I. and Toskovic, O. 2019. Connection between urban green areas and visitors' physical and mental well-being Urban Forestry \& Urban Greening. 40(4):299-307. 
Wang, X. 2009. Analysis of problems in urban green space system planning in China. J. Forest. Res. 20(1):79-82.

WCD. 1990. World Commission and Development. Our common future. Oxford University Press. Oxford, United Kingdom. 8 p.

WHO. 2017. World Health Organization. Urban green spaces: a brief for action. http://www.euro.who.int/_data/assets/pdf_file/0010/342289/Urban-green-spaces_en_who_ web3.pdf.

Xiao, D. X.; Dong, L.; Yan, H.; Yang, N. and Xiong, Y. 2018. The influence of the spatial characteristics of urban green space on the urban heat island effect in Suzhou Industrial Park. Sustainable Cities and Society. 40(5):429-439.

Yan, H.; Wu, F. and Dong L. 2018. Influence of a large urban park on the local urban thermal environment. Science of the Total Environment. 622-623:882-891. 